\title{
Relationship of the Shape of Subacromial Spur and Rotator Cuff Partial Thickness Tear
}

\author{
Young-Kyu Kim, Kyu-Hak Jung ${ }^{\circledR}$, Suk-Woong Kang ${ }^{1}$, Jin-Hun Hong, Ki-Yong Choi, Ji-Uk Choi \\ Department of Orthopedic Surgery, Gil Medical Center, Gachon University College of Medicine, Incheon, ${ }^{1}$ Department of Orthopedic Surgery, Pusan National \\ University Yangsan Hospital, Yangsan, Korea
}

Background: The present study was undertaken to evaluate the relationship between location of the rotator cuff tear and shape of the subacromial spur.

Methods: Totally, 80 consecutive patients who underwent arthroscopic repair for partial thickness rotator cuff tear were enrolled for the study. Bigliani's type of the acromion, type of subacromial spur, and location of partial thickness tear of the rotator cuff were evaluated using plain X-ray and magnetic resonance imaging. We then compared the groups of no spur with spur, and heel with traction spur.

Results: Of the 80 cases, 25 cases comprised the no spur group, and 55 cases comprised the spur group. There was a significant difference in type of tear $(p=0.0004)$ between these two groups. Bursal side tears were significantly greater (odds ratio $=6.000, p=0.0007)$ in the spur group. Subjects belonging to the spur group were further divided into heel (38 cases) and traction spur (17 cases). Comparing these two groups revealed significant differences only in the type of tear $(p=0.0001)$. Furthermore, the heel spur had significantly greater bursal side tear (odds ratio $=29.521, p=0.0005$ ) as compared to traction spur.

Conclusions: The heel spur is more associated to bursal side tear than the traction spur, whereas the traction spur associates greater to the articular side tear.

(Clin Shoulder Elbow 2019;22(3):139-145)

Key Words: Rotator cuff tears; Bone spur; Heel spur; Traction spur

\section{Introduction}

Various studies have reported the relationship between rotator cuff tear and subacromial spur. ${ }^{1-4)} \mathrm{Neer}^{5,6)}$ described the need for acromioplasty by stating that impingement of the rotator cuff with subacromial spur results in rotator cuff tear. However, since Codman $^{7)}$ stated that rotator cuff tear is the consequence of internal or degenerative changes, there have been several studies showing rotator cuff tear with altered rotator cuff degeneration in elderly patients. ${ }^{8,9)}$ The controversy over whether it is caused by collision with bony spurs or by internal degenerative factors still continues. Several studies suggest a correlation between the morphology of the subacromial spur and the rotator cuff tear., ${ }^{3,10-12)}$ Although still controversial whether the subacromial spur is the direct cause of rotator cuff tear according to the morphology, the direct relationship between the shape of the subacromial spur and the rotator cuff tear has raely been reported.

The authors hypothesize that site of the rotator cuff tear would differ in accordance with shape of the subacromial spur. Thus, the authors aimed to evaluate the relationship between location of the rotator cuff tear and shape of the subacromial spur.

\section{Methods}

\section{Patient Selection}

This was a longitudinal cross-sectional study approved by the

Received April 18, 2019. Revised August 4, 2019. Accepted August 4, 2019.

Correspondence to: Kyu-Hak Jung

Department of Orthopedic Surgery, Gil Medical Center, Gachon University College of Medicine, 21 Namdong-daero 774beon-gil, Namdong-gu, Incheon 21565, Korea

Tel: +82-32-460-3384, Fax: +82-32-423-3384, E-mail: jjangumom52@hanmail.net, ORCID: https://orcid.org/0000-0003-0211-8005

IRB approval: Gil Medical Center, Gachon University (No. GBIRB2014-329).

Financial support: None. Conflict of interests: None. 
Institutional Review Board at the Gil Medical Center, Gachon University (No. GBIRB2014-329). Between March 2012 and July 2014, 95 consecutive patients who underwent arthroscopic repair for partial thickness rotator cuff tears, operated by a single senior surgeon, were selected for the study. All patients had undergone totally 6 months conservative treatments. The preoperative magnetic resonance imaging (MRI) was examined for all cases, and arthroscopic evaluation confirmed the size of partial thickness rotator cuff tears. Cases selected for the final study included only those subjects with partial thickness rotator cuff tears of $40 \%$ to $70 \%$ on the bursal or articular side, based on the size of the footprint of the supraspinatus. Cases less than $40 \%$ partial thickness tear on MRI with no subsequent surgery were excluded from the study. Additionally, nearly full thickness tears of $70 \%$ or more were excluded, since it was difficult to clearly distinguish partial tear of only bursal or articular region. Since the intratendinous tear or severe tendinopathy on MRI is also difficult to perceive, it is theoretically necessary to perform a ballooning test with saline injection into the tendon to identify the definite intratendinous tear. Hence, subjects with intratendinous partial tears were also excluded, due to the vagueness of diagnosis itself. Lastly, definite traumatic rotator cuff tear and rotator cuff tear with severe osteoarthritis were also excluded. Sample sizes were calculated to detect a significant difference. Prior to this study, the authors conducted a pilot study with 20 cases. Spur was found in 13 cases. Among them, 8 cases were heel spur. The effect size was thus calculated as 0.33 . Accordingly, the sample size was determined to be 73 , and a total of 80 cases were finally confirmed by adding extra cases, considering the two-sided confidence of 95\% (type I error) and the power of $80 \%$. Thus, the entire study group consisted only of bursal or articular side partial thickness tears, and 80 cases were finally enrolled for this study. Of the total 80 cases, 41 cases (51.3\%) were male. The mean age was $58.0 \pm 8.0$ years; among total cases,

Table 1. Preoperative Demographic Data of Patients $(n=80)$

\begin{tabular}{lc}
\hline \multicolumn{1}{c}{ Variable } & Patient \\
\hline Sex (male) & $41(51.3)$ \\
Age (yr) & $58.0 \pm 8.0$ \\
Dominant hand & $51(63.8)$ \\
Right side & $43(53.8)$ \\
Type of tear (articular side/bursal side) & $26(32.5) / 54(67.5)$ \\
Type of spur (no spur/heel spur/traction spur) & $25(31.3) / 38(47.5) / 17(21.2)$ \\
Bigliani classification (I/II/III) $^{*}$ & $6(7.5) / 55(68.8) / 19(23.7)$
\end{tabular}

Values are presented as number (\%) or mean \pm standard deviation.

${ }^{\star}$ Type of tear was classified as articular or bursal side. ${ }^{\dagger}$ Type of spur was classified as no spur, heel spur, and traction spur. *Three distinct morphological shapes were sorted according to the Bigliani's classification; type I (flat), type II (curved), and type III (hooked). ${ }^{13)}$
51 cases $(63.8 \%)$ were dominant hands, and 43 cases (53.8\%) were right shoulders (Table 1). ${ }^{13)}$

\section{Radiologic Evaluation}

The Bigliani's type of the acromion was diagnosed by plain Xray. According to the Bigliani's classification, the type of acromion are classified as flat (type I), curved (type II), and hooked (type III) on the supraspinatus outlet view of shoulder radiographs. ${ }^{13)}$ We observed 6 cases (7.5\%) type I, 55 cases (68.8\%) type II, and 19 cases (23.7\%) type III (Table 1$)^{13)}$

All patients underwent preoperative MRI scans, performed using a 3.0-tesla scans (Skyra; Siemens Medical, Berlin, Germany). Oblique coronal, oblique sagittal, and axial T2-weighted fat-saturated images were acquired for assessment of the rotator cuff tendons. Slice thicknesses on the oblique coronal, oblique sagittal, and axial views were $3 \mathrm{~mm}, 4 \mathrm{~mm}$, and $3 \mathrm{~mm}$, respectively. The interslice gap on the coronal, sagittal, and axial views was $20 \%, 40 \%$, and $10 \%$, respectively. There were no cases of enhanced MRI. ${ }^{14)}$

The subacromial spur was evaluated on plain shoulder X-rays and shoulder MRIs, and classified as heel and traction spur. Heel spur is defined as a heel-like irregular bony protrusion located below the anterolateral side of the acromion. True anteroposterior view and supraspinatus outlet view of plain X-ray, and oblique coronal and sagittal image of MRI revealed thickened bony structures with irregular shapes, which were subsequently confirmed by arthroscopic findings (Fig. 1). Traction spur is defined as an anterior protruding bony structure running along the coracoacromial ligament from the anterolateral corner of the acromion. The short and sharp bony protrusions were confirmed on the true anteroposterior view of plain X-ray and oblique coronal image of MRI. Furthermore, bird-beak like protruding bony structures along the coracoacromial ligament from beneath the acromion were identified on supraspinatus outlet view of plain X-ray and sagittal image of MRI. The final diagnosis was made in arthroscopic findings (Fig. 2). In some cases, it was difficult to accurately classify the shape of the spur by X-ray; hence, final classification was done by considering all findings from $X$ ray, $\mathrm{MRI}$, and arthroscopy. Bony sclerotic changes and minimal bony irregularity on the X-ray and MRI, with no indication of any prominent bony project, were considered to have no subacromial spur. In the current study enrolling 80 cases of acromion, subacromial spur was observed in 55 cases (68.7\%), of which heel spur and traction spur accounted for 38 cases $(47.5 \%)$ and 17 cases $(21.2 \%)$, respectively (Table 1$).{ }^{13)}$

The location and the size of the rotator cuff tear were classified as articular and bursal side tear by oblique coronal MRIs. These findings were subsequently confirmed by arthroscopic examination. In this study, we confirmed 26 cases (32.5\%) having articular side tear, and 54 cases (67.5\%) with bursal side tear (Table 1). ${ }^{13)}$ 

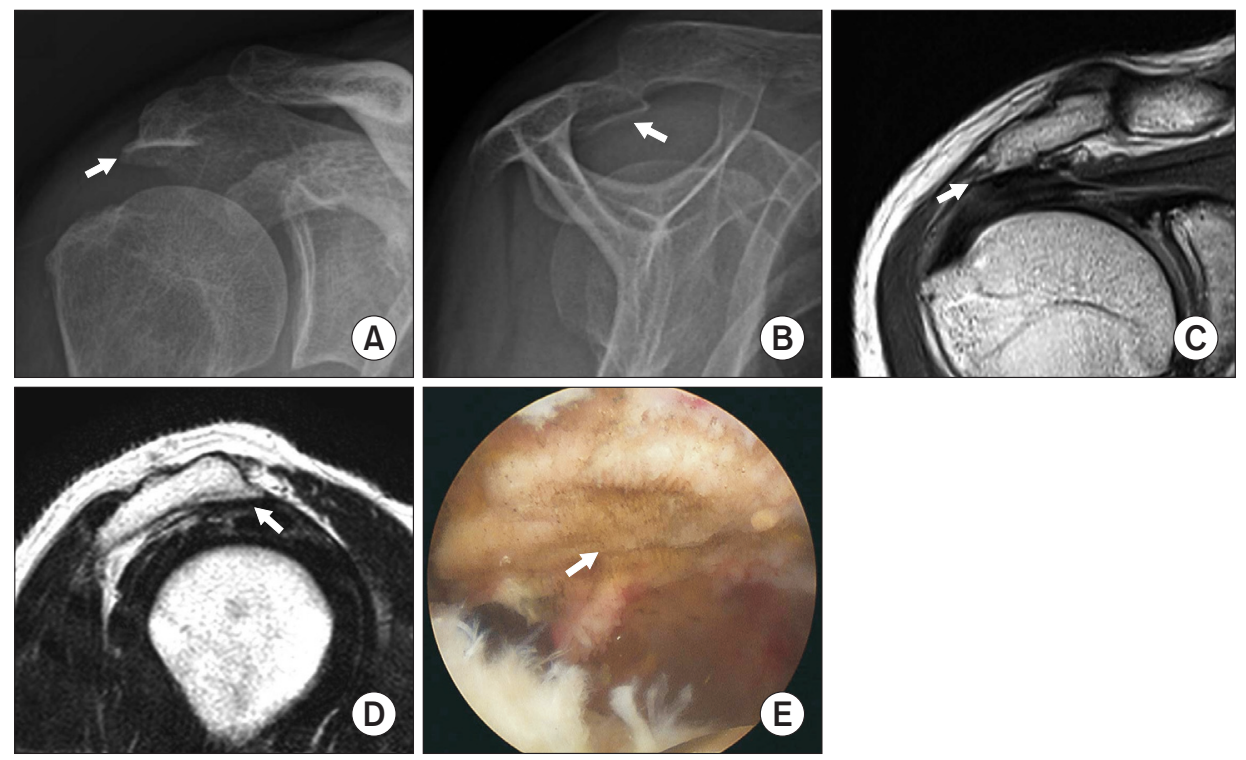

Fig. 1. Plain X-ray, magnetic resonance images, and arthroscopic image of heel spur. True anterior-posterior view (A), supraspinatus outlet view (B), oblique coronal view (C), oblique sagittal view (D), and arthroscopic view (E) (white arrows).
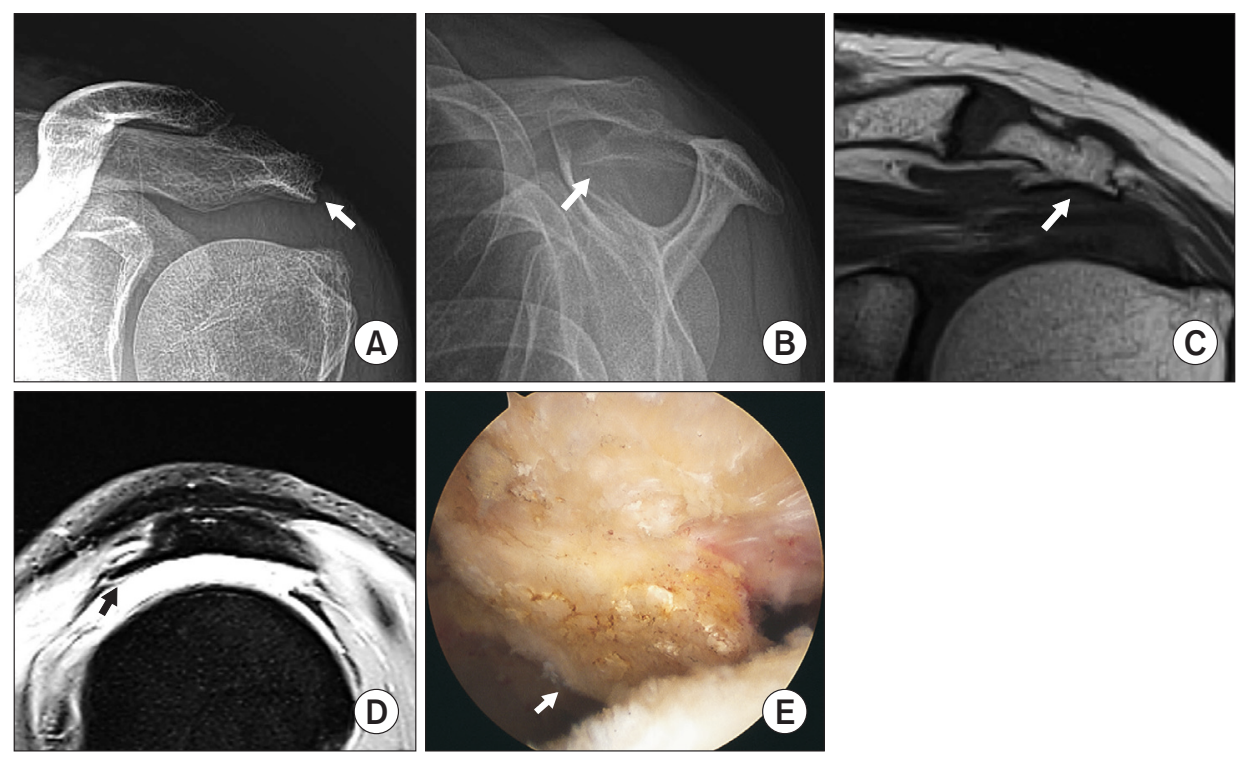

Fig. 2. Plain X-ray, magnetic resonance images, and arthroscopic image of traction spur. True anterior-posterior view (A), supraspinatus outlet view (B), oblique coronal view $(C)$, oblique sagittal view $(D)$, and arthroscopic view (E) (white or black arrows).

\section{Statistical Analysis}

For comparing the no spur and spur groups, age was analyzed with student's t-test, and sex, dominant hand, right or left side, type of tear, and Bigliani's type of acromion were analyzed using chi-squared test. However, in several variables, more than $20 \%$ of the expected counts of each cell on the contingency table were less than 5. For those cases, we applied the Fisher's exact test. In cases that the data table was larger than $2 \times 2$, the Freeman-Halton extension of the Fisher's exact test was used, instead of the traditional Fisher's exact test. ${ }^{15)}$ Univariate binary logistic regression analysis was used to analyze the effect of spur on the bursal side rotator cuff tears. Multivariable logistic regression analysis evaluated the effect of spur on the bursal side rotator cuff tears, according to the all independent variables, using

the backward elimination procedure. The condition for the inclusion of variables was $0.05 .^{14)}$

For comparing the heel spur and traction spur groups, age was analyzed with Mann-Whitney U-test as a non-parametric test (since size of samples was only 17), whereas sex, dominant hand, right or left side, type of tear, and Bigliani's type of acromion were analyzed by the chi-squared test. Fisher's exact test and the Freeman-Halton extension of the Fisher's exact test were similarly adopted to the variables, as above. Analysis of covariance (ANCOVA) is the most appropriate method if random sampling is not performed, and data with unclear causality is taken into consideration for various covariances that may affect the outcome. In this study, however, logistic regression was performed since data were identified as non-normal and non- 
equivalent data. Univariate logistic regression analysis was used to analyze the effect of heel spur on the bursal side rotator cuff tears. Multivariable logistic regression analysis was used to evaluate the effect of heel spur on the bursal side rotator cuff tears, similar to the procedure described above.

All radiologic and arthroscopic assessments were performed by two board-certified orthopedic surgeons having more than 5 years' experience. In case of differing interobserver categorization, the final decision was made by a senior orthopedic surgeon who did not participate in the measurements. Cohen's kappa was calculated as a measure of agreement. According to Landis and Koch, ${ }^{16)}$ the following ratings for the interpretation of kappa were used: poor (0.20), fair (0.21-0.40), moderate (0.41-0.60), good (0.61-0.80), and excellent (0.81-1.00). However, a kappa value less than 0.5 was usually estimated as lower reliability. In this study, the intra-observer and inter-observer coefficients were $0.86(\mathrm{Cl}, 0.82-0.91)$ and $0.75(\mathrm{Cl}, 0.69-0.80)$, respectively. All statistical tests are 2-tailed, and $p<0.05$ is considered statistically significant. The SAS software package (ver. 9.4 TS Level 1M3; SAS Institute, Cary, NC, USA) was used as a statistical analyzer.

\section{Results}

Of the 80 cases considered in the final analysis, 25 cases were identified as the no spur group, and 55 cases as spur group. Significant differences were observed in the comparison of dominant hand $(p=0.0482)$ and type of tear $(p=0.0004)$. When considering type of tear, the no spur group had a greater number of articular side tears, whereas the spur group had more bursal side tears. Bigliani's classification did not differ according to spur type (Table 2). ${ }^{13)}$ Univariate and multivariable logistic regression were used to identify factors affecting the bursal side tear. In both analyses, the presence or absence of spur was the only significant factor, and the odds ratio for the effect of spur to bursal side tear as compared to no spur was significantly 6.000 (95\% confidence interval, 2.126-16.936; $p=0.0007$ ) (Table 3 ). Thus, univariate and multivariable logistic regression were performed with dependent variables. Likewise, significant result was obtained only in the tear variable; i.e., only tear and spur variables showed strong correlation.

The spur group was further divided into cases with heel spur (38 cases) and traction spur (17 cases). Comparisons between the two sub-groups showed significant differences only in type of tear $(p=0.0001)$. In the heel spur group, the number of cases with bursal side tear was significantly higher than the articular side tear, whereas articular side tear was more frequent in the traction spur group (Table 4). ${ }^{13)}$ Univariate and multivariable logistic regression were subsequently used to identify factors affecting bursal side tear in the two groups. Similar to the previous data, only type of spur was affected by both analyses. The odds ratio of heel spur to bursal side tear was significantly 29.521 (95\% confidence interval, 4.450-195.851; $p=0.0005)$, when compared to traction spur (Table 5).

\section{Discussion}

This study was undertaken to determine the relationship between shape of the subacromial spur and partial thickness tear of rotator cuff, and to analyze the relationship between the

Table 3. Significant Factors for Bursal Side Rotator Cuff Tears by Univariate and Multivariate Logistic Regression Analysis between No Spur and Spur Groups*

\begin{tabular}{cccc}
\hline Variable & $p$-value & Exp (B) & 95\% CI \\
\hline No spur or spur & & & \\
Univariate analysis & 0.0007 & 6.000 & $2.126-16.936$ \\
Multivariate analysis & 0.0007 & 6.000 & $2.126-16.936$ \\
\hline
\end{tabular}

Exp (B): exponentiation of the B coefficient, CI: confidence interval.

${ }^{*}$ Multivariate binary logistic regression analysis was performed, using the backward elimination procedure. The condition of selection stay for variables was 0.05 . ${ }^{\dagger} \operatorname{Exp}(\mathrm{B})$ means odds ratio. ${ }^{*}$ No spur was reference category. The results of univariate and multivariate were the same. If only one variable is consistently significant, multivariate analysis may show the same results as univariate analysis. In other words, except for variable of spur or not, there were no other variables showing significant results from the beginning of the analysis, and this situation persisted throughout the entire backward elimination process.

Table 2. Comparison between No Spur and Spur Groups

\begin{tabular}{lccc}
\hline \multicolumn{1}{c}{ Variable } & No spur $(\mathrm{n}=25)$ & Spur $(\mathrm{n}=55)$ & $p$-value \\
\hline Sex (male) & $13(52.0)$ & $28(50.9)$ & $0.9279^{*}$ \\
\hline Age $(\mathrm{yr})$ & $58.6 \pm 6.0$ & $57.6 \pm 8.8$ & $0.5539^{\dagger}$ \\
\hline Dominant hand & $12(48.0)$ & $39(70.9)$ & $0.0482^{*}$ \\
Right side & $10(40.0)$ & $33(60.0)$ & $0.0963^{*}$ \\
\hline Type of tear (articular side/bursal side) & $15(60.0) / 10(40.0)$ & $11(20.0) / 44(80.0)$ & $0.0004^{*}$ \\
Bigliani's classification $(\mathrm{I} / \mathrm{II} / \mathrm{III})^{\varsigma}$ & $3(12.0) / 17(68.0) / 5(20.0)$ & $3(5.5) / 38(69.1) / 14(25.5)$ & $0.5281^{\|}$ \\
\hline
\end{tabular}

Values are presented as number (\%) or mean \pm standard deviation.

${ }^{*}$ Chi-squared test. ${ }^{\dagger}$ Student t-test. ${ }^{*}$ Type of tear was classified as articular or bursal side. ${ }^{5}$ Three distinct morphological shapes were sorted according to the Bigliani's classification; type I (flat), type II (curved), and type III (hooked). ${ }^{13)}$ "Freeman-Halton extension of the Fisher's exact test. 
Table 4. Comparison between the Groups of Heel and Traction Spur

\begin{tabular}{lccc}
\hline \multicolumn{1}{c}{ Variable } & Heel Spur $(\mathrm{n}=38)$ & Traction Spur $(\mathrm{n}=17)$ & $\mathrm{p}$-value \\
\hline Sex (male) & $17(44.7)$ & $11(64.7)$ & $0.1710^{*}$ \\
Age $(\mathrm{yr})$ & $58.6 \pm 6.0$ & $57.6 \pm 8.8$ & $0.5539^{\dagger}$ \\
\hline Dominant hand & $26(68.4)$ & $13(76.5)$ & $0.7497^{*}$ \\
Right side & $23(60.5)$ & $10(58.8)$ & $0.9052^{*}$ \\
Type of tear (articular side/bursal side) & $2(5.3) / 36(94.7)$ & $9(52.9) / 8(47.1)$ & $0.0001^{*}$ \\
Bigliani's classification (I/II/III) & $3(7.9) / 26(68.4) / 9(23.7)$ & $0(0) / 12(70.6) / 5(29.4)$ & $0.6883^{\|}$ \\
\hline
\end{tabular}

Values are presented as number (\%) or mean \pm standard deviation.

${ }^{*}$ Chi-squared test. ${ }^{\dagger}$ Student $\mathrm{t}$-test. ${ }^{*}$ Type of tear was classified as articular or bursal side. ${ }^{5}$ Three distinct morphological shapes were sorted according to the Bigliani classification; type I (flat), type II (curved), and type III (hooked). ${ }^{13)}$ "Freeman-Halton extension of the Fisher's exact test.

Table 5. Significant Factors for Bursal Side Rotator Cuff Tears by Univariate and Multivariate Logistic Regression Analysis between the Groups of Heel and Traction Spur*

\begin{tabular}{cccc}
\hline Variable & $p$-value & $\operatorname{Exp}(\mathrm{B})^{\dagger}$ & $95 \%$ CI \\
\hline Heel or traction spur & & & \\
Univariate analysis & 0.0006 & 20.250 & $3.651-112.297$ \\
Multivariate analysis & 0.0005 & 29.521 & $4.450-195.851$ \\
\hline
\end{tabular}

Exp (B): exponentiation of the B coefficient, CI: confidence interval.

${ }^{\star}$ Multivariate binary logistic regression analysis was performed, using the backward elimination procedure. The condition of selection stay for variables was $0.05 .{ }^{\dagger} \operatorname{Exp}(\mathrm{B})$ means odds ratio. ${ }^{*}$ Traction type spur was reference category.

two variables. Considering prior studies, the authors analyzed the spur by categorizing the cases as heel spur and traction spur. Oh et al. ${ }^{11)}$ categorized subacromial spurs into six different categories, and reported that full thickness rotator cuff tears were present in the heel spur group. Similarly, Tucker and Snyder ${ }^{12)}$ reported that heel spur was a risk factor for bursal side partial thickness tears and full thickness rotator cuff tears. On the other hand, Ogawa et al. ${ }^{10)}$ and Hamid et al. ${ }^{3)}$ reported that traction spur of $5 \mathrm{~mm}$ or more were frequent in full thickness rotator cuff tear. In a recent study examining the relationship among the heel type osteophyte, critical shoulder angle and rotator cuff tears, Kim et al. ${ }^{17}$ showed that rotator cuff tear was affected more by heel type osteophytes than critical shoulder angle. They proposed that heel type osteophytes could be one of the factors related to induce rotator cuff tears. Conversely, another study by Sasiponganan et al. ${ }^{18)}$ reported that subacromial spur, including heel type and traction type, was associated only with subscapular tear among rotator cuff tear. However, this study had its limitation, as it did not analyze according to the type of spur.

Chambler et al. ${ }^{19)}$ reported an increase in osteocyte enzyme activity at the origin of the coracoacromial ligament in the presence of rotator cuff tear, and secondary subacromial spur formation in the presence of rotator cuff tear. In their study, the shape of the subacromial spur was not concretely described, but was a characteristic of the traction spur formed at the origin of the coracoacromial ligament. In addition, Hashimoto et al. ${ }^{20)}$ reported that degenerative changes of the articular side, accompanied by microinjury of the rotator cuff, were the main causes of rotator cuff tear in pathologic studies. Several other studies have emphasized the intrinsic and degenerative causes of rotator cuff tears in relation to the generation of subacromial spurs. ${ }^{5,21-23)}$ In the current study, a bursal side tear was predominant in heel spurs, but articular side tears were more frequent in traction spurs, suggesting a positive correlation between degenerative rotator cuff tear and articular side tear.

Contrarily, Neer ${ }^{5)}$ introduced the impingement syndrome, and reported that rotator cuff tear was induced with progression in mechanical compression within subacromial bursitis and subacromial spur. Fu et al. ${ }^{24)}$ emphasized the extrinsic factor, describing the rotator cuff tear induced by the direct impingement of the coracoacromial arch of the supraspinatus and the secondary impingement caused by the micromotion of the forward elevation exercise in overhead throwing athletes. Considering that most of the tears found in heel type spur was bursal sided in the present study, suggests that impingement by heel spur may result in a tear.

Since the current study is cross-sectional, causal relationships according to the time order cannot be confirmed. However, articular side tear was common in the no spur group, and bursal side tear was confirmed in the spur group. This suggests that in the progression of rotator cuff tear, the articular side tear might precede degeneration, and gradually lead to development of the subacromial traction spur. The purpose of the present study was to evaluate whether rotator cuff tear was initiated from either articular or bursal side before the tear was converted to full thickness, and the factors associated with cuff tear. Undoubtedly, the above assumptions cannot be clarified in the present study, and need to be elucidated through additional studies in the future.

Rotator cuff repair is accompanied by acromioplasty in many cases. In the current study, location of the cuff tear differs according to the type of subacromial spur, and may be the basis of 
selective treatment of acromioplasty. Oh et al. ${ }^{25)}$ reported pain and functional evaluation of bursal side partial thickness tear after surgery. They showed that most patients had heel spurs, and recommended that rotator cuff repair with acromioplasty should be performed early due to the high probability of progressing to full thickness tear. However, numerous other studies have shown that acromioplasty is not clinically important, and there is no great merit. ${ }^{26-28)}$ Therefore, the authors believe that acromioplasty should be performed selectively, although preventive acromioplasty is required to prevent the progression to full thickness tear of the bursal side tear due to heel spur.

There are limitations in the present study. First, there is no gold standard classification for subacromial spur, except for the Bigliani's classification for the shape of acromion. Even Bigliani's classification, a theory delineating the relationship between acromial morphology and impingement of the rotator cuff tear, has not been established. ${ }^{10)}$ Therefore, a more complementary, long-term and large-scale study is required, to be recognized as an objective classification of the currently available classification method. Second, there are radiological limitations. Peh et al. ${ }^{29)}$ reported that even if performed by a skilled radiologist, the image may look different if the angle of view is slightly changed, which may eventually change the classification of the subacromial spur. Finally, in this study, the incidence of articular side tear was lower than that of previously reported studies. It is considered that this is due to the fact that asymptomatic partial tear was excluded, since it applied to patients who underwent surgery during symptomatic partial thickness tears.

\section{Conclusion}

The heel spur is more associated with bursal side tear than the traction spur, and the traction spur is related to the articular side tear. However, it is difficult to determine the chronological relationship between traction spur and articular side tear.

\section{References}

1. Bonsell S, Pearsall AW 4th, Heitman RJ, Helms CA, Major NM, Speer KP. The relationship of age, gender, and degenerative changes observed on radiographs of the shoulder in asymptomatic individuals. J Bone Joint Surg Br. 2000;82(8):1135-9.

2. Gill TJ, Mclrvin E, Kocher MS, Homa K, Mair SD, Hawkins RJ. The relative importance of acromial morphology and age with respect to rotator cuff pathology. J Shoulder Elbow Surg. 2002;11(4):327-30. doi: 10.1067/mse.2002.124425.

3. Hamid N, Omid R, Yamaguchi K, Steger-May K, Stobbs G, Keener JD. Relationship of radiographic acromial characteristics and rotator cuff disease: a prospective investigation of clinical, radiographic, and sonographic findings. J Shoulder Elbow Surg. 2012;21(10):1289-98. doi: 10.1016/j.jse.2011.09.028.
4. Panni AS, Milano G, Lucania L, Fabbriciani C, Logroscino CA. Histological analysis of the coracoacromial arch: correlation between age-related changes and rotator cuff tears. Arthroscopy. 1996;12(5):531-40. doi: 10.1016/S0749-8063(96)901905.

5. Neer CS 2nd. Anterior acromioplasty for the chronic impingement syndrome in the shoulder: a preliminary report. J Bone Joint Surg Am. 1972;54(1):41-50.

6. Neer CS 2nd. Impingement lesions. Clin Orthop Relat Res. 1983;(173):70-7.

7. Codman EA. The shoulder: rupture of the supraspinatus tendon and other lesions in or about the subacromial bursa. Boston (MA): Thomas Todd; 1934. 67-79.

8. Tempelhof S, Rupp S, Seil R. Age-related prevalence of rotator cuff tears in asymptomatic shoulders. J Shoulder Elbow Surg. 1999;8(4):296-9. doi: 10.1016/S1058-2746(99)90148-9.

9. Uhthoff HK, Sarkar K; American Academy of Orthopedic Surgeons. The effect of aging on the soft tissues of the shoulder. Paper presented at: The Shoulder: A Balance of Mobility and Stability Workshop; 1992 Sep; Vail, USA. p.269-278.

10. Ogawa K, Yoshida A, Inokuchi W, Naniwa T. Acromial spur: relationship to aging and morphologic changes in the rotator cuff. J Shoulder Elbow Surg. 2005;14(6):591-8. doi: 10.1016/ j.jse.2005.03.007.

11. Oh JH, Kim JY, Lee HK, Choi JA. Classification and clinical significance of acromial spur in rotator cuff tear: heeltype spur and rotator cuff tear. Clin Orthop Relat Res. 2010;468(6):1542-50. doi: 10.1007/s11999-009-1058-5.

12. Tucker TJ, Snyder SJ. The keeled acromion: an aggressive acromial variant--a series of 20 patients with associated rotator cuff tears. Arthroscopy. 2004;20(7):744-53. doi: 10.1016/j.arthro. 2004.06.018.

13. Bigliani LU, Morrison DS, April EW. The morphology of the acromion and rotator cuff impingement. Orthop Trans. 1986;10:228-35.

14. Kim YK, Jung KH, Kim JW, Kim US, Hwang DH. Factors affecting rotator cuff integrity after arthroscopic repair for medium-sized or larger cuff tears: a retrospective cohort study. J Shoulder Elbow Surg. 2018;27(6):1012-20. doi: 10.1016/ j.jse.2017.11.016.

15. Kim YK, Jung KH, Park CK, Yun SB. Morphologic factors related to repair outcomes for delaminated rotator cuff tears: a minimum 2-year retrospective comparison study. Arthroscopy. 2019;35(2):332-40. doi: 10.1016/j.arthro.2018.08.040.

16. Landis JR, Koch GG. The measurement of observer agreement for categorical data. Biometrics. 1977;33(1):159-74.

17. Kim JH, Min YK, Gwak HC, Kim CW, Lee CR, Lee SJ. Rotator cuff tear incidence association with critical shoulder angle and subacromial osteophytes. J Shoulder Elbow Surg. 2019;28(3):470-5. doi: 10.1016/j.jse.2018.08.026.

18. Sasiponganan C, Dessouky R, Ashikyan O, et al. Subacromial 
impingement anatomy and its association with rotator cuff pathology in women: radiograph and MRI correlation, a retrospective evaluation. Skeletal Radiol. 2019;48(5):781-90. doi: 10.1007/s00256-018-3096-0.

19. Chambler AF, Pitsillides AA, Emery RJ. Acromial spur formation in patients with rotator cuff tears. J Shoulder Elbow Surg. 2003;12(4):314-21. doi: 10.1016/mse.2003. S1058274603000302.

20. Hashimoto T, Nobuhara K, Hamada T. Pathologic evidence of degeneration as a primary cause of rotator cuff tear. Clin Orthop Relat Res. 2003;(415):111-20. doi: 10.1097/01.blo. 0000092974.12414 .22$.

21. Chansky HA, lannotti JP. The vascularity of the rotator cuff. Clin Sports Med. 1991;10(4):807-22.

22. Nixon JE, DiStefano V. Ruptures of the rotator cuff. Orthop Clin North Am. 1975;6(2):423-47.

23. Ozaki J, Fujimoto S, Nakagawa Y, Masuhara K, Tamai S. Tears of the rotator cuff of the shoulder associated with pathological changes in the acromion. A study in cadavera. J Bone Joint Surg Am. 1988;70(8):1224-30.

24. Fu FH, Harner CD, Klein AH. Shoulder impingement syndrome. A critical review. Clin Orthop Relat Res. 1991;(269): 162-73.
25. Oh JH, Oh $\mathrm{CH}$, Kim $\mathrm{SH}$, Kim JH, Yoon JP, Jung JH. Clinical features of partial anterior bursal-sided supraspinatus tendon (PABST) lesions. J Shoulder Elbow Surg. 2012;21(3):295-303. doi: 10.1016/j.jse.2011.07.025.

26. Beard DJ, Rees JL, Cook JA, et al.; CSAW Study Group. Arthroscopic subacromial decompression for subacromial shoulder pain (CSAW): a multicentre, pragmatic, parallel group, placebo-controlled, three-group, randomised surgical trial. Lancet. 2018;391(10118):329-38. doi: 10.1016/S01406736(17)32457-1.

27. Bond EC, Maher A, Hunt L, et al. The role of acromioplasty when repairing rotator cuff tears-no difference in pain or functional outcome at 24 months in a cohort of 2,441 patients. $\mathrm{N}$ Z Med J. 2017;130(1458):13-20.

28. Kolk A, Thomassen BJW, Hund H, et al. Does acromioplasty result in favorable clinical and radiologic outcomes in the management of chronic subacromial pain syndrome? A doubleblinded randomized clinical trial with 9 to 14 years' follow-up. J Shoulder Elbow Surg. 2017;26(8):1407-15. doi: 10.1016/ j.jse.2017.03.021.

29. Peh WC, Farmer TH, Totty WG. Acromial arch shape: assessment with MR imaging. Radiology. 1995;195(2):501-5. doi: 10.1148/radiology.195.2.7724774. 